\title{
Positioning and Synchronization of Industrial Robots
}

\author{
Christoph Herrmann*, M. Hennes*, M. Juretzko*, C. Munzinger**, M. Schneider** \\ * Karlsruhe Institute of Technology, Geodetic Institute, Karlsruhe, Germany. Email: christoph.herrmann@kit.edu \\ ** Karlsruhe Institute of Technology, Institute of Production Science, Karlsruhe, Germany. Email: \\ munzinger@wbk.uka.de
}

\begin{abstract}
This paper presents experiments and their results to position industrial robots and to evaluate the synchronization of co-operating robots using laser trackers.
\end{abstract}

Keywords - Laser Tracker, T-Probe, Cross Correlation, Trigger

\section{INTRODUCTION}

Within the collaborative research center SFB TR10 a process chain for the flexible production and machining of extruded aluminum profiles is developed and put into practice. The SFB TR10 is a co-operation of the wbk Institute of Production Science of the Karlsruhe Institute of Technology (KIT), the University Munich and the University Dortmund. The process chain includes in the subproject A4 co-operating robots for fully automated guiding and handling of the profiles. To guarantee the correct form of the extruded profile the robots have to be precisely aligned with it. Therefore their tool center points (TCP) have to be known exactly. A method is presented, which delivers the TCP using 6DOF (6 Degrees of Freedom) equipment. Besides the accuracy of the robot's position the synchronization of co-operating robots is of high interest. The experimental set-up for investigating the robot's synchronization and results are shown.

\section{MEASUREMENT TOOLS}

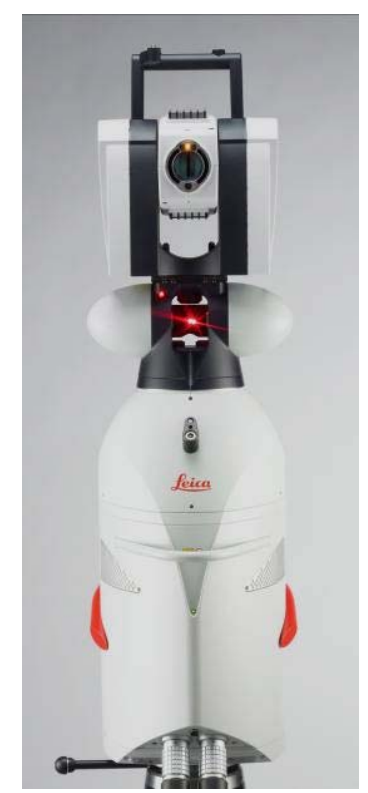

Figure 1. AT901 with T-Cam.
For the accurate surveying of the robots the Geodetic Institute of KIT (GIK) used two of Leica's laser trackers the LTD 500 and the Absolute Tracker AT901 in combination with T-Cam and T-Probe. The T-products determine position as well as orientation (6DOF) of an object, which is equipped with the T-Probe. The T-Probe incorporates a Corner Cube Reflector (CCR) and IRdiodes. The position is measured conventionally by the laser tracker. Furthermore, a camera which is mounted on top of the AT901 (T-Cam, Fig. 1, [www.leicageosystems.com]) takes images of the T-Probe's IRdiodes. Photogrammetric methods in the calculation routine analyze the spatial distribution of the IR-diodes and determine the three orientation angles of the T-Probe. According to the measurement task different styli can be attached to the probe to determine surface points.

The GIK used the T-Probe to determine the position of the robot's tool adapter. The T-Probe allows to determine $6 \mathrm{DOF}$ and is easily attached to the robot's tool. With a calibration routine described in section III.A it was possible to measure the TCP and align it with the aluminum profile.

For determining the synchronization of the robots the T-Probe could not be used, because the LTD 500 does not enable 6DOF-measurements. Each robot was equipped with a cateye reflector instead. These reflectors provide a much wider aperture angle than the standard CCRs and ensure that the laser beam would not break during the measurements. The cateyes were attached to magnetic reflector holders which were hot glued to the robot's tool. Section III.B explains the measurements to determine the synchronization in detail.

\section{PROCEDURES AND RESULTS}

\section{A. Positioning}

All measurements were carried out in a coordinate system based on the extruding press. The front of the press (Fig. 2) defines the X-Z-plane of the coordinate system. Several drift nests (Fig. 3) for the 1.5 inch CCR widely distributed around the press served as the system's realization and as basis for all measurements. They ensure the possibility of a transformation between data acquired from different laser trackers and/or laser tracker positions. 


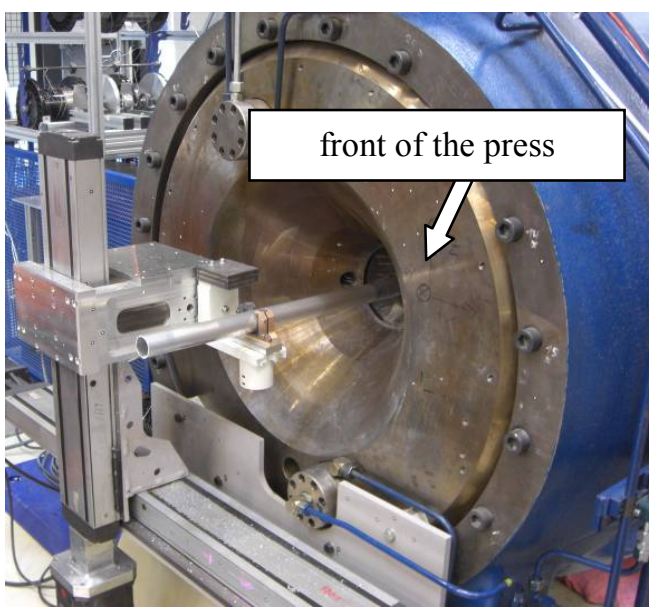

Figure 2. Front of the press.

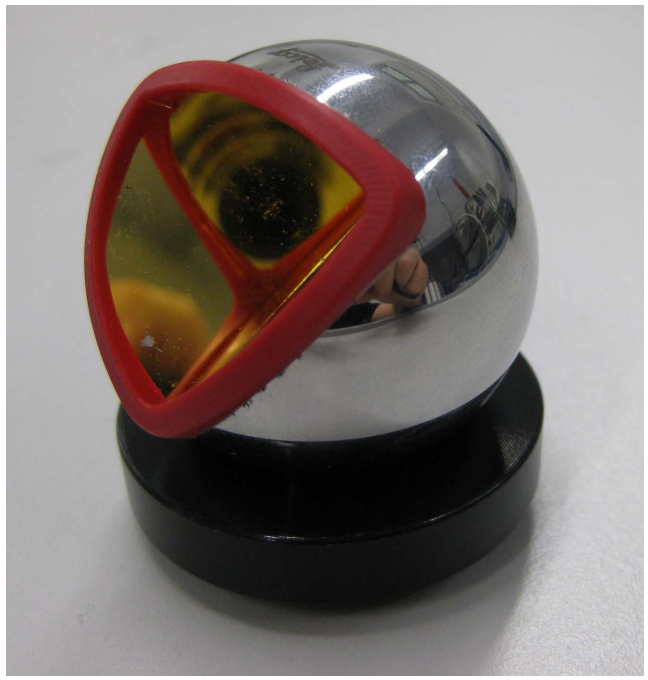

Figure 3. Drift nest with 1.5-inch CCR.

The goal was to align the robot's tool exactly with the center of the extruded aluminum profile. To reach that goal it was necessary to determine the tool center point of each tool. Unfortunately, it was not possible to physically measure the actual TCP with tactile or reflector-less measuring methods. Nevertheless, the task was fulfilled with the help of Leica's 6DOF measurement device TProbe.

The T-Probe was attached to the tool and a calibration routine determined the probe's tip in the coordinate system of the probe itself. The calibration routine is included in Leica's tracker programming interface emScon and is normally used to calibrate the different styli that can be attached to the T-Probe. The routine determines the T-Probe's tip coordinates throughout a number of movements of the T-Probe with the tip held in the exact same position. The idea was to define the TCP as the tip of the T-Probe. Consequently, the T-Probe equipped robot moved with its TCP held in the same position. The result of this procedure was a virtual stylus definition with the (virtual) tip of the T-Probe being the actual TCP. Hence, every measurement with this virtual stylus delivers the actual position of the TCP. The accuracy of the tip's definition is influenced by the measurement accuracy of the T-Probe and the accuracy of the robot's movements during the calibration routine. The accuracy of the final tip definition was approximately $0.2 \mathrm{~mm}$. By accurately determining the TCP it was possible to align the robot and the tool, respectively, with the center of the extruded aluminum profile. The center of the profile was determined by measuring a cone around the profile and calculating its axis. The routine has to be repeated for each robot individually.

\section{B. Synchronization}

For determining the synchronization (i.e. - possibly variable - latency) between the two robots triggered measurements to both robots at the same time were carried out. Each tracker observed one robot. The robots' tools moved along a $500 \mathrm{~mm}$ long straight line parallel to the $\mathrm{Y}$-axis of the coordinate system. The measurement setup is depicted in Fig. 4 with R1 and R2 being the tools of the two robots. One measurement cycle contained the movement from 0 to $500 \mathrm{~mm}$, a short stop and the movement back to $0 \mathrm{~mm}$. To ensure fully synchronized data, the robot control unit provided a trigger signal for the trackers with a frequency of $500 \mathrm{~Hz}$, i.e. the trackers measured a point every two milliseconds. The trigger signal ensured the trackers to start the measurement at the same point of time without any delay. This is crucial for investigating the synchronization of two objects, in this case, the two robots. To ensure occurring delays between the robots are not caused by the measurement itself, the effects of a clock drift were investigated in [5]. These experiments showed a slight drift, but as long as the trackers are controlled by a trigger, i.e. every single measurement is activated by the trigger and not by the tracker's own clock, the clock drift is insignificant. Consequently the trigger should not only provide the start signal for the measurement of each cycle but also activate every single measurement within a cycle with the above mentioned frequency.

The nominal values for the robot tracks were given to the robot control unit by a real-time numerical control (NC) as a list with every position in space. The coordinates referred to the robot coordinate system and the $\mathrm{X}$ and $\mathrm{Z}$ coordinates of all the points had the value zero. The time interval of the nominal values in the list is $4 \mathrm{~ms}$. The NC provides the robot control unit every $12 \mathrm{~ms}$ with a new nominal position [5]. In order to determine a delay between the robots, the measuring frequency had to be at least $250 \mathrm{~Hz}$. With the above mentioned frequency of $500 \mathrm{~Hz}$ this requirement was met. To evaluate the differences between the nominal tracks and the measured tracks the sampling rate of the measured tracks was decreased, i.e. the measured data was thinned out.

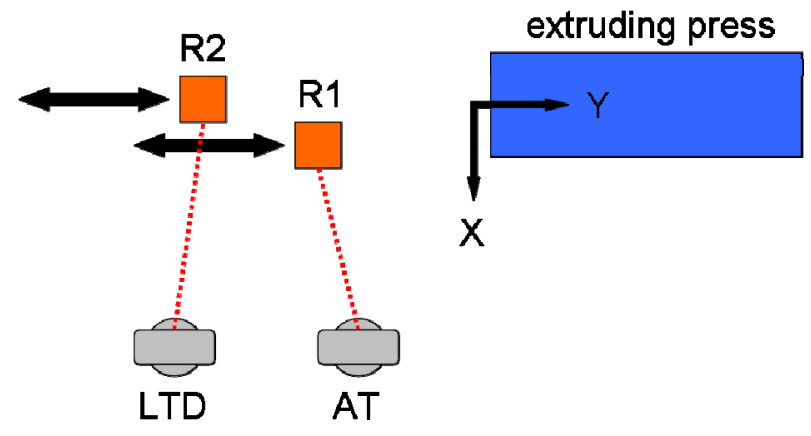

Figure 4. Layout of the measurement setup. 
This investigation could not be done with respect of $6 \mathrm{DOF}$, because there was only one 6DOF-System available. Each robot was equipped with a cateye reflector instead of the T-Probe (Fig. 5). Because of the robots moving along a straight line parallel to the $\mathrm{Y}$-axis of the coordinate system the reference to the actual TCP, which could not be maintained throughout the change of reflectors, was no longer necessary. The entire tool moved only in one coordinate direction with the limits of the robot's accuracy. Therefore the position of each cateye on the tool could be chosen with respect to free sight to the laser tracker. However, using the T-Probe, the method can be applied to orientation values as well.

The tracker measurements deliver ASCII-files with the coordinates $\mathrm{X}, \mathrm{Y}$ and $\mathrm{Z}$ in the tracker coordinate system and a timestamp for each coordinate triple. The first step for evaluating the data was the transformation of the coordinates into the system of the extruding press with a six-parameter Helmert-transformation.

Although the robots as well as the trackers are oriented in the same coordinate system, the definition of the tracks has only the same orientation, but not the same origin. The starting point of each track was chosen randomly and set as the origin in the robot's control unit. I.e. each robot moved the tool from the position $(0,0,0)$ to $(0,500,0)$ in the robot's system and back. The laser trackers however register the coordinates of the tool in the superior system of the extruding press. Hence, in this system the starting points of the robots are different. Nevertheless, with the above mentioned premise of the same orientation of both the robots and the tracker's coordinate system, the comparison of the measured tracks and the nominal tracks is possible. By a simple offset correction the tracks received the same origin. I.e. the coordinates of the measured tracks were reduced by the value of each starting point. That value is the mean of the first 50 coordinate triples of each track while the robots stayed at the starting point.

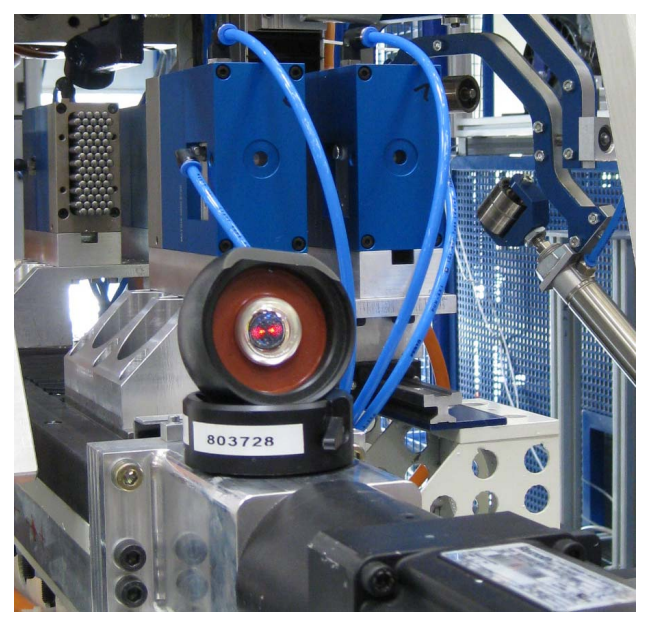

Figure 5. Cateye reflector.

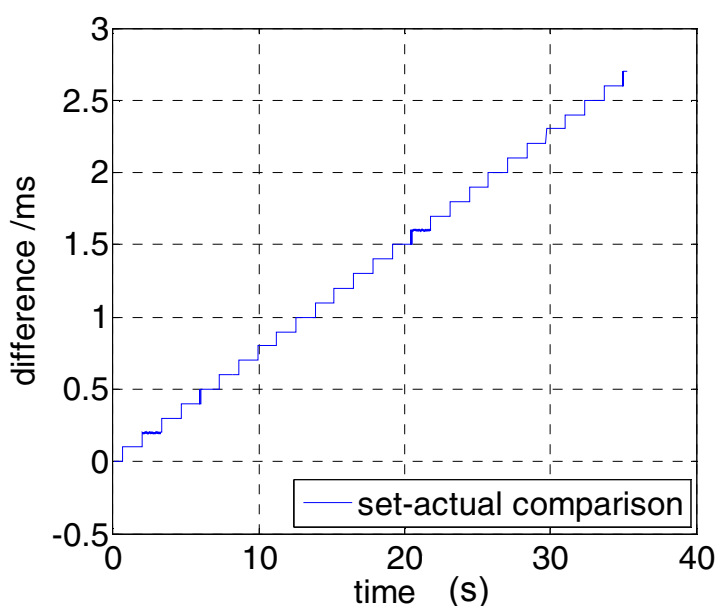

Figure 6. Oscillator drift.

Comparing a nominal time scale with an increment of two milliseconds with the actual time scale of the trigger signal for the trackers a drift as depicted in Fig. 6 is obvious. The drift is $2.5 \mathrm{~ms}$ over $35 \mathrm{~s}$. Because of the used trigger signal as described above, the drift is insignificant.

It is of high importance that the point in time when the robots start moving is exactly the same, i.e. that they are fully synchronized. So, the delay between the robots has to be derived precisely as it is a crucial parameter for operating two autonomous robots in co-operation. A cross correlation of the two measured tracks (time series) of one measurement cycle provides this information. Because of the minimal movements of the robots perpendicular to the direction of motion (see Fig. 10) only the Y-coordinates of the tracks were used for the cross correlation. The cross correlation coefficient is a function of the time lag between the measured tracks. In terms of signal processing it is calculated with (1). Fig. 7 depicts the cross correlation coefficient against the time lag. The position of the function's maximum equates the time displacement (delay) between the tracks. This method allows determining the time displacement with the resolution of the measuring frequency. In this case the resolution is $2 \mathrm{~ms}$ because the original $500 \mathrm{~Hz}$ data was used.

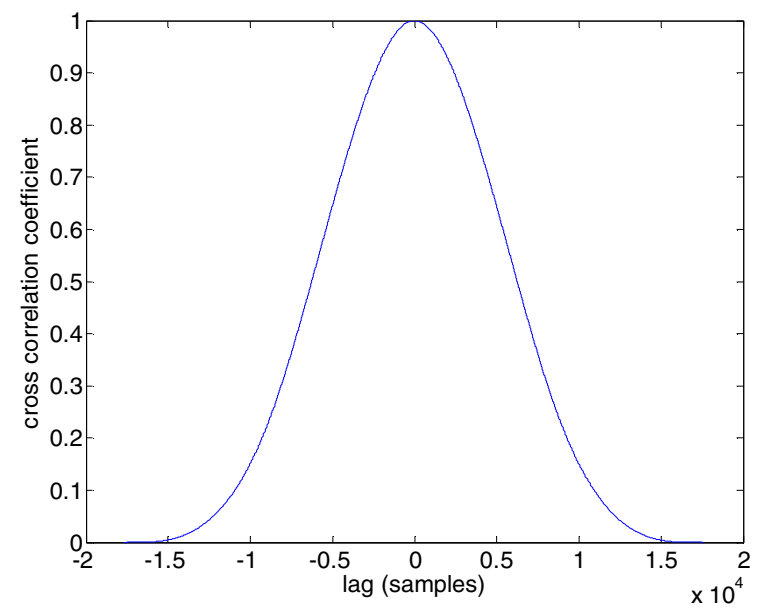

Figure 7. Cross correlation function between the two tracks of one measurement cycle. 


$$
R(\tau)=\int_{-\infty}^{\infty} Y_{1}(t) Y_{2}(t+\tau) d t
$$

$$
\begin{aligned}
& \text { with } R \quad \text { cross correlation coefficient } \\
& \tau \quad \text { time lag } \\
& Y_{1}(t), Y_{2}(t) \text { time series, in this case the } \\
& \text { tracks of R1 and R2 }
\end{aligned}
$$

Evaluating the position of the cross correlation function's maximum with respect to the time lag displays a delay of three samples (i.e. $6 \mathrm{~ms}$ ) between the two robots. The maximum of the function is rather even due to the measuring frequency. I.e. because of the high measuring frequency in relation to the relatively slow movement of the robots, the difference between two measured points is small. Consequently the cross correlation leads to quite similar cross correlation coefficients for two close by time lags. In order to get a sharper definition of the maximum of the correlation function, one would have to decrease the measuring frequency. But that would also decrease the timeresolution for determining the delay and is therefore out of the question.

The comparison of the measured tracks provides the result as depicted in Fig. 8. It shows the difference between the entire tracks of cycle 1 of R1 and cycle 1 of $\mathrm{R} 2$, cycle 2 of R1 and cycle 2 of R2 etc. The diagram displays the high repeatability of the robots movements. The difference of the measured tracks between the cycles is $0.1 \mathrm{~mm}$ at most. Furthermore, the diagram shows the robots move in the same manner. I.e. they accelerate and break equally fast and maintain the same speed. The diagram also tells that the robot $\mathrm{R} 2$ which was observed by the LTD 500 lags behind the robot R1 with the above mentioned delay. One can see that because of the change of sign of the difference after the turn point (at $18 \mathrm{~s}$ ) of the track. Theoretically the differences should be equally distributed with the same displacement to zero. The offset of about $0.2 \mathrm{~mm}$ could not be explained and will be investigated further.

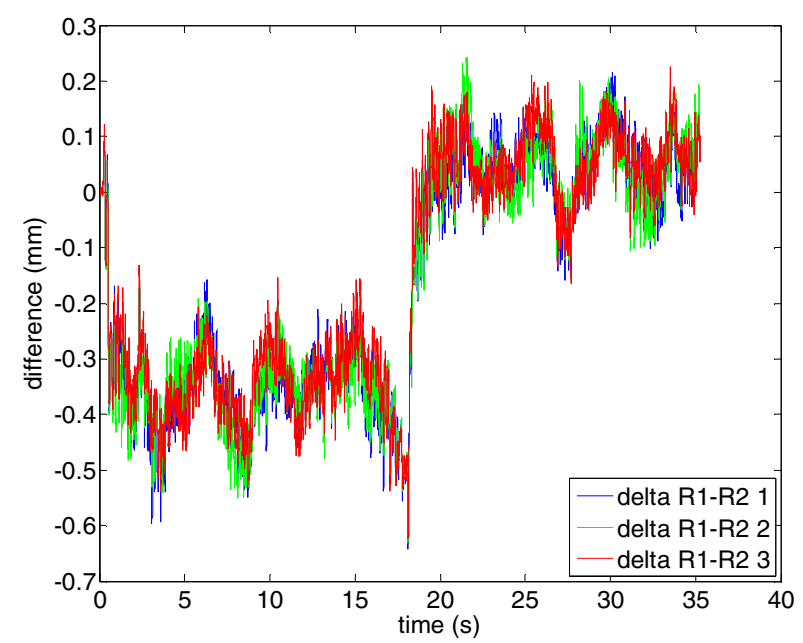

Figure 8. difference of the measured tracks per measurement cycle
Although the measurement was activated by a trigger signal, the actual point of time on the time scale of the robot control unit when the trackers started the measurement could not be retrieved precisely. Due to issues concerning the recording of the data, there was a time offset between the starting point of the robot's movement and the starting of the laser tracker measurement. Nevertheless, for comparing the measured tracks with the nominal track the same routine using the cross correlation function was used. Of each measured track and the nominal track the cross correlation was calculated to determine the time displacement between the tracks. Comparing the nominal with the measured tracks displays the robots absolute accuracy. Fig. 9 and Fig. 10 show the result of this comparison. In Fig. 9 the differences to the nominal track in the direction of motion (parallel to the Y-axis of the coordinate system) are depicted for each robot and each track individually. Due to reaction time and contouring error the actual and nominal position differ up to $4 \mathrm{~mm}$. The problem of contouring errors is well known but cannot be eliminated entirely. As long as the robots maintain the same contouring error, the co-operating robots will work perfectly together. Crucial are varying contouring errors, which are detected as described above.

Concerning the other two components (X- and Zcoordinate) of the movement perpendicular to the direction of motion the maximum difference to the nominal track is $0.65 \mathrm{~mm}$ (see Fig. 10) in the Z-coordinate of robot 1 . The standard deviations for the differences to the nominal track for robot 1 are $0.09 \mathrm{~mm}$ in $Z$ and $0.07 \mathrm{~mm}$ in $X$. For robot 2 they are $0.08 \mathrm{~mm}$ in $Z$ and $0.03 \mathrm{~mm}$ in $X$. The jumps as well in $X$ as in $Z$ occur at the same time (at ca. $6 \mathrm{~s}$ and $32 \mathrm{~s}$ ) and are caused by the movement of the robot's joints and actuators.

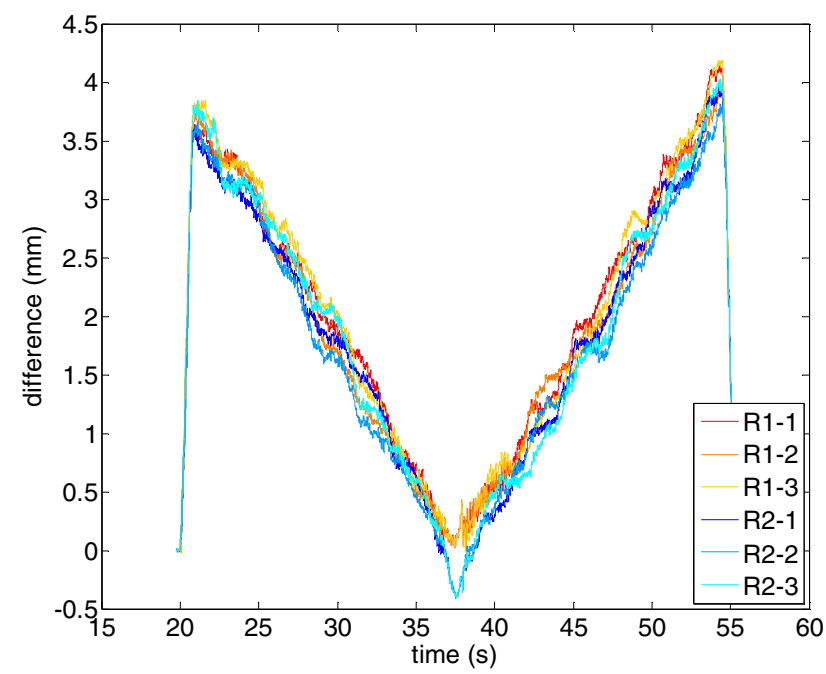

Figure 9. differences in $\mathrm{Y}$ to the nominal track 

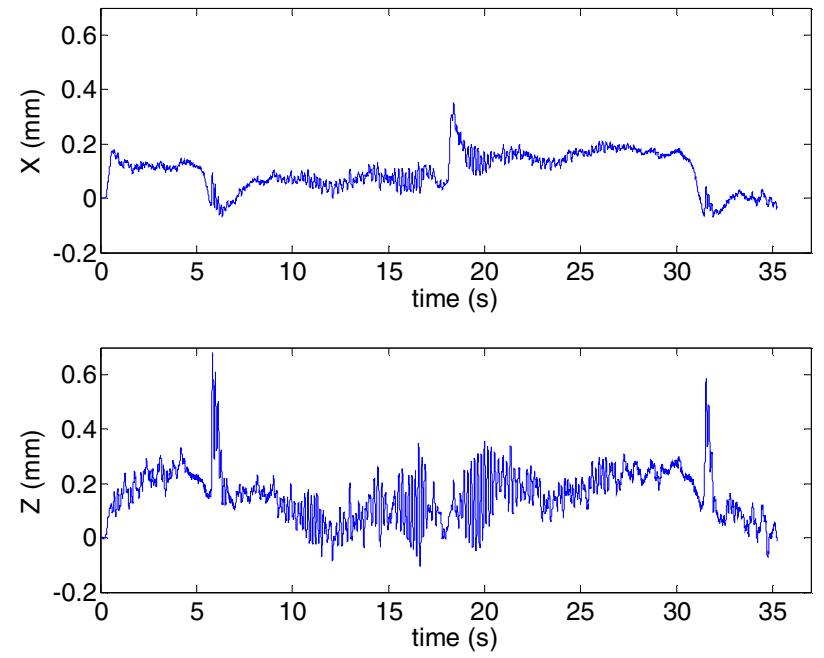

Figure 10. differences in $\mathrm{X}$ and $\mathrm{Z}$ to the nominal track

\section{CONCLUSION}

The tip-calibration procedure of Leica's T-Probe can be efficiently used for determining the TCP of a robot. The investigation shows potential for further developments with the aim of automating the process. This procedure has the edge over the often time consuming and complicated methods of positioning a robot and does not require the T-Mac.

Furthermore, the paper depicts the ability of laser trackers to act on a trigger pulse. Consequently, they are predestined for spatio-temporal surveys of co-operating robots. The presented method allows analyzing the synchronization of co-operating robots and has the potential of improving the quality of process chains and their products.

This paper is based on scientific work within the collaborative research center SFB TR10 funded by the "Deutsche Forschungsgemeinschaft" (DFG)

\section{REFERENCES}

[1] Depenthal, C. (2008): „A Time-referenced 4D Calibration System for Kinematic Optical Measuring Systems. Proc. of $1^{\text {st }}$ International Conference on Machine Control and Guidance, June 24-26 2008, ETH Zurich, CH.

[2] DEPENTHAL, C., BARTH, M. (2007): „Zur Leistungsfähigkeit eines zeitreferenzierten Dreharms als Prüfmittel für 4D-Messsysteme in Hochgeschwindigkeitsanwendungen“, AVN, Heft 7/2007 p. 244249.

[3] Fleischer, J.; Munzinger, C.; Stengel, G. (2006): "Flying Cutting of Spatially Curved Extrusion Profiles", In: Advanced Materials Research: Flexible Manufacture of Lightweight Frame Structures, Band 10 (2006), p. 35-42.

[4] JURETZKO, M. (2007): „Untersuchungen zur Wiederholgenauigkeit eines geregelten Winkelmesssystems am Beispiel eines Lasertrackers LTD 500“, Beitrag zum 15. Internationalen Ingenieurvermessungskurs, Graz, 17.-20.4.2007, in: Brunner, F. K. (Hrsg.), Ingenieurvermessung 07, Herbert Wichmann Verlag, Heidelberg, p. 181-186.

[5] JuRetzko, M., HenNeS, M. (2008): „Monitoring of the spatiotemporal movement of an industrial robot using a laser tracker", Proc. of $1^{\text {st }}$ International Conference on Machine Control and Guidance, June 24-26 2008, ETH Zurich, CH, p. 315-320. 